\title{
Powder and Solvent for Suspension for Injection in Multidose Container
}

National Cancer Institute

\section{Source}

National Cancer Institute. Powder and Solvent for Suspension for Injection in Multidose

Container. NCI Thesaurus. Code C153578.

Medicinal product consisting of a powder and solvent for suspension for injection presented in a multidose container; usually the powder is presented in the multidose container while the solvent is presented in a separate container, and is added to the powder in order to prepare the suspension for injection in the multidose container. 